\title{
EL LUGAR DE LA POLITICA DENTRO DE LA TRIPARTICION DE LA PHILOSOPHIA PRACTICA ANTES DE LA RECEPCION MEDIEVAL DE LA POLITICA DE ARISTOTELES
}

Francisco Bertelloni**

SÍNTESE - É de todos conhecida a influência exercida por Aristóteles no desenvolvimento do pensamento político medieval. Procura-se aqui fazer uma análise histórica, filológica e filosófica do momento anterior a 1265 - data da tradução da Politica - para mostrar como aquele momento influenciou a maneira como a obra foi recebida no Ocidente.

PALAVRAS-CHAVE - Aristóteles, política, filosofia medieval.
ABSTRACT - Everyone knows Aristotle's influence on the development of the political ideas in the Middle Ages. The author tries here to make an historical, philological and philosophical analysis of the time previous to the year 1265 - when the translation of Politics appeared - in order to show how this moment has influenced on the manner of the reception of this work in the west.

KEY WORDS - Aristotle, politics, medieval philosophy.

Actualmente está fuera de duda la influencia ejercida por la Politica de Aristóteles en el desarrollo del pensamiento político a partir de la segunda mitad del siglo XIII. Esa influencia no solamente se manifestó a través de la aparición de numerosos comentarios ${ }^{1}$ a la Politica, sino especialmente en la redacción de tratados

* Este trabajo fue reahizado con el apoyo de un subsidio UBACYT de la Universidad de Buenos Aires.

** Universidad de Buenos Aires.

1 En relación con los comentarios es pionero el artículo de Martin Grabmann, Die mittelalterlichen Kommentare zur Politik des Aristoteles (Sitzungsberichte der Bayerischen Akademie der Wissenschaften, Phil.-hist. Abteilung, Bd. I, Heft 10, 1941), Munich, 1941, esp. p. 8/27. La más reciente puesta al día del problema ha sido realizada por Christoph Flüeler en Rezeption und Interpretation der Aristotelischen Politica im späten Mittelalter, 2 ts., Amsterdam - Philadelphia, 1992.

\begin{tabular}{l|l|l|l|l|l|} 
VERTTAS & Porto Alegre & v. 43 & $\mathrm{n}^{0} 3$ & Setembro 1998 & p. 563-576
\end{tabular}


políticos de inspiración aristotélica. ${ }^{2}$ No es mi intención ocuparme aquí de la influencia que ejerció la Politica en el pensamiento político medieval. Solo me interesa hacer algunas reflexiones de carácter histórico-filosófico sobre un momento de la historia intelectual de la edad media que es cronológicamente anterior a la recepción de la Politica y que, como momento preparatorio, parece haber ejercido una fuerte influencia en el proceso de recepción de ese texto aristotélico.

El tema de la preparación de la recepción de la Politica no es nuevo. Ya algunos estudiosos de la teoría política medieval han afirmado que esa recepción tuvo lugar porque fue precedida por una suerte de preparación de la recepción y que esa preparación fue especialmente favorecida por la tradición de las divisiones philosophiae surgidas en el medio intelectual de la Facultad de Artes de Paris de la primera mitad del siglo XIII. Más concretamente, atribuyen un papel protagónico en la recepción de la Politica al esquema tripartito de la philosophia practica que forma parte de esas divisiones philosophiae y que divide a la philosophia practica en ethica, oeconomica y politica. ${ }^{3}$ Parece legítimo preguntar, pues, porqué ese esquema tripartito de la philosophia practica fue tan importante en la recepción del texto de la Politica.

Si dejamos de lado momentáneamente la Politica y analizamos el proceso de recepción de todos los textos aristotélicos en la edad media como fenómeno unitario, es necesario considerar especialmente un aspecto de ese fenómeno para entender ese proceso de recepción: se trata del ingreso progresivo y gradual en Occidente de los textos aristotélicos a partir de principios del siglo XII y del modo como esos nuevos textos iban completando y llenando el esquema del saber científico expresado a través de las divisiones philosophiae, es decir, expresado a través de la nueva expresión del saber científico que reemplazaba el viejo esquema de las artes liberales.

Aunque en el esquema de las divisiones philosophiae los textos aristotélicos recientemente conocidos iban llenando de nuevo contenido las distintas ramas del conocimiento, de todos modos siempre quedaban ramas de saber vacias,i.e. ramas con nombre pero sin contenido, o lo que es lo mismo, sin textos que pudieran satisfacer esa rama del conocimiento en términos científicos. De alli que las divisiones philosophiae fueran esquemas del saber completos como esquemas, pero muchas veces truncos e incompletos en cuanto a su contenido, pero por ello mismo constituían un estímulo para seguir buscando los textos que faltaban. En cierto modo, esos esquemas constituían una suerte de plan o proyecto de búsqueda, de modo que a medida que esos textos eran encontrados, iban completando el contenido del esquema del saber científico. ${ }^{4}$

2 Sobre los tratados véase, tambien de Martin Grabmann, Studien über den Einfluß der aristotelischen Philosophie auf die mittelalterlichen Theorien über das Verhältnis von Kirche und Staat (Sitzungsberichte der Bayerischen Akademie der Wissenschaften, Phil.- hist. Abteilung), Munich, 1934.

3 Véase por ejemplo, Chr. Flüeler, Rezeption und Interpretation der Aristotelischen Politica im späten Mittelalter, Teil I, Amsterdam-Philadelphia, 1992, p. 3.

4 La tesis es de Charles Lohr, quien la resume asi: "By the middle of the twelfth century these younger masters had come to realise that there were whole areas of knowledge of which they knew only the names. It was only natural that they should try to learn more of their subject matter" (cfr. "The medieval interpretation on Aristotle", en: The Cambridge History of Later Me- 
Precisamente,el caso de la politica como rama de la philosophia practica, era uno de los casos de disciplinas que, con nombre, pero sin contenido definido, integraban el esquema del saber científico expresado por la divisio philosophia. En efecto, la politica venía formando parte de la philosophia practica desde el siglo V, sin que se lograra definir con certeza el contenido científico que le correspondía. Así sucedió hasta que el texto de la Politica de Aristóteles fue conocído y traducido en forma completa por Guillermo de Moerbecke en $1265 .{ }^{5}$ Cuando el texto de la Politica fue traducido, la politica como rama del conocimiento encontró en ese tratado un texto que le permitía legitimarse como conocimiento científico. Recién entonces esa disciplina - que entre el siglo $\mathrm{V}$ y el siglo XIII solo había tenido un espacio ganado en cuanto al nombre, pero cuyo contenido era muy difuso - se incorporó con aplomo al esquema del saber científico y ganó su espacio como parte del sistema teórico de disciplinas unificadas en el sistema teórico de la nueva philosophia practica.

El objetivo de esta comunicación es reconstruir los detalles del proceso mediante el cual el texto de la Politica se incorporó al esquema del saber identificándose con la disciplina "politica". En rigor, esa identificación constituyó un fenómeno paradójico de la historia de la filosofía. En efecto, aunque injustificadamente se insiste en hablar de "tripartición aristotélica" en la edad media, el esquema tripartito de la philosophia practica dentro del que fue incorporado el texto de la Politica en 1265 no fue un esquema que la edad media hubiera recibido de la tradición aristotélica, sino que era de origen neoplatónico; por la otra, ese esquema de origen neoplatónico fue confirmado como esquema, i.e. en su estructura formal y llenado con un nuevo contenido específicamente aristotélico cuando fueron conocidos en forma completa los llamados libri morales de Aristóteles en 1265.

La explicación de esa paradoja histórica debe ser desplegada haciendo referencia a algunos problemas implícitos en ella: el primero es histórico, el segundo es filológico y el tercero es filosófico-sistemático.

El problema histórico se refiere, por una parte, al origen histórico de la división tripartita de la philosophia practica que dominó en Occidente hasta inmediatamente antes de la completa recepción occidental de los libri morales de Aristóteles, y por la otra y a la evolución histórica de esa división tripartita hasta 1265. Ello invita a realizar una reconstrucción de la historia del modelo teórico presentado por el esquema de la philosophia practica que dominó en Occidente hasta inmediatamente antes de la recepción del texto de la Politica. Objetivo de esa reconstrucción es poner de manifiesto las diferencias de filiación histórica que separan esa tradición de la philosophia practica anterior a 1265 del esquema de la philosophia practica que irrumpe en Occidente recién en 1265, cuando tiene lugar el pleno conocimiento de los libri morales de Aristóteles.

dieval Philosophy, Cambridge, 1982, p. 83). El texto de Lohr es recomendable para una correcta inteligencia del proceso de recepción de los textos aristotélicos en el medioevo.

5

Más detalles acerca de la recepción de la Politica véase Chr. Flüeler, "Die Rezeption der 'Politica' des Aristoteles an der Pariser Artistenfakultät im 13. und 14. Jahrhundert”, en: Miethke, J. (ed.), Das Publikum politischer Theorie im 14. Jahrhundert, München, 1992, p. 127/138. 
El problema filológico concierne al vocabulario de la politica, i.e. a la politica como término que formaba parte del vocabulario científico: ¿qué era la politica dentro del esquema de la philosophia practica en cuanto al nombre "politica" y cómo se justificaba la utilización de ese término antes de que fuera conocido el texto de la Politica traducido por Guillermo de Moerbecke? Es decir: ¿cómo se constituye y genera el nombre de la disciplina llamada "politica"?

El problema específicamente filosófico tiene que ver con el lugar teórico atribuído a la politica como disciplina científica dentro del tríptico de la philosophia practica antes y đespués de la completa recepción de los libri morales. En este caso se debe caracterizar el difuso contenido teórico de la politica dentro de la estructura sistemática de la philosophia practica hasta 1265 y se lo debe comparar con el modo como la politica estaba teóricamente vinculada con las otras ramas de la philosophia practica dentro del sistema de la división tripartita elaborado por Aristóteles y conocido en 1265. Esa comparación debe establecer las similitudes y diferencias teórico-doctrinales entre la división transmitida por la tradición neoplatónica y la específicamente aristotélica.

He individualizado cada uno de estos problemas a efectos de que pueda percibirse el amplio alcance y las distintas dimensiones implícitas en el tema. Limitaciones de espacio y de tiempo me impiden tratar aquí en detalle y en particular cada uno de los tres problemas que he mencionado. Cada uno de ellos aparecerá entrelazado con los otros a lo largo de una presentación de carácter predominantemente histórico del problema que reconstruiré en lo que sigue.

Esa reconstrucción debe comenzar llamando la atención sobre la existencia de una tendencia a colocar en Aristóteles el origen histórico de la tripartición de la philosophia practica en ethica individualis, oeconomica y politica. Debe tenerse en cuenta, sin embargo, que esa tripartición aparece, antes de Aristóteles, en el Protágoras de Platón, 318e: oikeía (lo propio), oikía (la casa), pólis (la ciudad).

A partir de esa formulación, la tripartición de la philosophia practica hace una carrera triunfal. Aunque Aristóteles no fue ni el primero que formuló la tripartición ni el autor de la formulación literaria que divide la filosofía moral del modo como lo hace esa tripartición, ${ }^{6}$ sí fue Aristóteles el autor de la primera sistematización teórica de las relaciones entre politica, oeconomica y ethica, cuyas características pueden ser colegidas de la Ethica Nicomachea y de la Politica.

La primera de ellas aparece en la Politica. Aristóteles caracteriza cada una de las partes de la philosophia practica como ámbitos en los que se verifican relaciones de autoridad y subordinación entre un sujeto que manda y domina y otro que obedece. ${ }^{7}$ Esas relaciones de subordinación penetran tan radicalmente cada una de las partes de la moral aristotélica, que ellas incluso aparecen en la parte de la philosophia practica en la que el vínculo de subordinación parece más difícil de

6 La formulación literaria de la tripartición no es aristotélica. El pasaje de Eth. NiC., VI, 1142a 9 solo sugiere esa división: "Quamvis forte non est hoc 'bonum individuale' sine oeconomica, neque sine urbanitate 'i.e. sine politica'". Pero este pasaje no fue conocido antes de $1246 / 7$, i.e. antes de la traducción de toda la Ethica Nicomachea. Igualmente en la Ethica Eudemia, 1218b 13 aparece la distinción entre politiké, oikonomiké y phrónesis, pero este tratado era igualmente desconocido.

7 Politica, 1252a 6 ss. 
establecer, es decir en la ethica individual, en la que el alma debe gobernar al cuerpo y el intelecto al apetito. ${ }^{8}$

En segundo lugar Aristóteles marca diferencias cualitativas entre la ethica individual, la oeconomica y la politica: el vínculo que relaciona al individuo consigo mismo en la ethica no es el mismo que lo relaciona con los miembros de la casa en la oeconomica o con los ciudadanos de la civitas en la politica.Estas diferencias no solo resultan de la distancia que separa al buen hombre del buen ciudadano - y que separan a la ethica de la politica ${ }^{9}$ - o de la distancia entre el gobierno de la casa y el gobierno politicum..$^{10}$ Además de ello Aristóteles subraya las dificultades implícitas en la justificación teórica del tránsito desde cada una a las otras partes de la philosophia practica. Es el caso que se da al final de la Ethica Nicomachea, cuando urgido por la necesidad de llevar a la práctica el discurso teórico de la ethica (individual), debe fundamentar el paso desde la ethica individual a la politica. Allí se refiere a la impotencia de los vínculos paternales, vigentes en la oeconomica, para hacer efectivos los planteos teóricos de la ethica ${ }^{11}$ que deben concretarse en la politica. Entonces recurre a la doctrina de la ley, como parte de la politica ${ }^{12}$ y concluye que la politica debe utilizar la coactividad de la ley para persuadir, por medio de esa coactividad, a quienes no se dejan persuadir por las palabras. ${ }^{13}$

Por último fundamenta la primacía lógico-ontológica de la politica sobre la ethica y sobre la oeconomica como equivalente a la primacía del todo sobre las partes, y lo hace afirmando que la politica es polo de absorción de los fines de las otras dos partes de la philosophia practica. A su vez, aunque en la ethica y la oeconomica no se verifican aún relaciones específicamente políticas, sin embargo

8 "Est quidem sicut dicimus primum in animaii contemplari et despoticum principatum et politicum. Anima quidem corpori enim dominatur despotico principatu, intellectus enim apetitui politico et regali. In quibus manifestum esi, quod secundum naturam et expediens corpori regi à anima, et passibili parti ab intellectu, et parte rationem habente; ex aequo autem aut e contrario, nocivum omnibus.Iterum in homine et in aliis animalibus similiter se habet..." (ibid., 1254b 1 - 10).

"Bonum autem virum dicimus esse secundum virtutem perfectam. Quod quidem igitur contingit civem existentem studiosum non possidere virtutem secundum quam est studiosus vir, manifestum" (ibid., 1276b 27 - 30).

10 "Quicumque igitur quidem existimant politicum et regalem et oeconomicum et despoticum, idem non bene dicunt" (ibid., 1252a 6 ss.).

11 "Paterna quidem igitur praeceptio non habet forte neque coactivum, neque totaliter quae unius viri, nisi regis existentis, vel alicuius talis" (Eth. Nic., 1180a 18-20).

12 "Leges autem politicis operibus assimilantur" (Eth. Nic., 1181b 1).

13

"Si utique est aliquis finis operabilium, quem propter seipsum volumus...: manifestum quoniam hic utique erit bonus, et optimus... Si autem sic,tentandum est figuraliter accipere illud quid quidem est, et cuius discipinarum aut virtutum. Videbitur autem utique principalissime et maxime architectonicae esse. Talis utique et civilis apparet...Utente vero hac, reliquis practicis disciplinis, amplius autem legem proponente quid oportet operari, et a quibus abstinere; huiusmodi finis complectitur utique eos qui aliarum. Quapropter hic utique erit humanum bonum" (Eth. Nic.,1094a 22 - b 6); "Dicamus autem resumentes, quoniam omnis cognitio et electio, bonum aliquid desiderat, quid est hoc, quod dicimus civilem desiderare" (ibid., 1095a 14 - 15); "Quoniam omnem civitatem videmus communitate quamdam existentem, et omnem communitatem boni alicuius institutam; eius enim quod videtur boni gratia, omnia operantur omnes: manifestum quod omnis bonum aliquod coniecturat. Maxime autem principalissimum omnium maxime principalis, et omnes alias circumplectens. Haec autem est quae vocatur civitas, et communicatio politica" (Politica, 1252a 1 $6)$. 
la politica como ciencia arquitectónica se sirve de ellas para efectivizar los fines de la civitas. ${ }^{14}$ Por ello, durante el proceso de formación de las relaciones propias del individuo en la ethica y de los miembros de la casa en la oeconomica, esas dos partes adquieren una dimensión política pues tienden a la constitución de la civitas como comunidad perfecta. Esa primacía de la politica otorga unidad a las tres partes de la philosophia practica y supera el aislamiento entre ellas. La unidad de la philosophia practica pasa por la politicidad, que es asumida por las otras ciencias prácticas gracias a la fuerza congiutinante de la politica.

Entre Aristóteles y la antigüedad tardía la tripartición realiza un periplo interesante. Después de Aristóteles, Filón de Alejandría vuelve a hacer referencia a oeconomica y politica. ${ }^{15}$ Arius Didymus retoma la tripartición en ethica, oeconomica y politica. ${ }^{16}$ Séneca distingue entre moralis, oikonomiké y civilis. ${ }^{17} \mathrm{~A}$ partir del siglo II, la tripartición vuelve a aparecer en el platonismo medio: Alkinoos [Albinos?] por ejemplo,habla explícitamente de tres ciencias prácticas, ethikón, oikonomikón y politikón. ${ }^{18}$ Diógenes Laercio, en sus Vitae philosophorum, atribuye la tripartición as Aristóteles. ${ }^{19} \mathrm{El}$ vocabulario de Calcidio referido a la tripartición es moralis, domestica y publica. ${ }^{20}$ En el siglo $\mathrm{V}$ la tripartición reaparece en el comentador neoplatónico de Aristóteles Ammonio, ${ }^{21}$ y en el siglo VI en Eliae, ${ }^{22}$ quienes la dividen en tres disciplinas prácticas ue llaman ethikón, oikonomikón y politikón.

La politica como parte de la philosophia practica ingresa por primera vez en el mundo latino a través de Boecio. A partir de Boecio el esquema de la división tripartita se impone hasta Hugo de San Victor en el siglo XII, es decir durante siete siglos. Sin embargo, la información que recibe el mundo latino acerca de la philosophia practica a partir de Boecio no es homogénea. Entre Boecio y Hugo de San Victor es posible distinguir dos tradiciones similares, pero independientes entre sí.

La primera línea,inaugurada por Boecio,articula la philosophia practica en una triplex divisio: la primera parte trata de la cura sui con el de objeto aumentar la

14 "Lex autem coactivam habet potentiam, sermo ens ab aliqua prudentia et intellectu" (Eth. NiC., 1180a $18-22)$.

15 Stoicorum veterum fragmenta, collegit Ioannes ab Arnim, vol. II, Stuttgart, 1964, p. 209.

16 Ioannis Stobaei Anthologium, recensuit Curtius Wachsmuth, Berolini, 1884, Lib. II, p. 148.

17 "Philosophiae tres partes esse dixerunt et maximi et plurimi auctores: moralem, naturalem, rationalem [...] Quidam ex Peripateticis quartam partem adiecerunt civilem [...] Quidam adiecerunt his partem, quam oikonomikén vocant" (cfr. Sénèque, Lettre à Lucilius, t. IV, Paris, 1962, 22 s.).

18 Alcinoos, Enseignement des doctrines de Platon, Introduction, texte établie et commenté par John Whittaker et traduit par Pierre Louis, Paris, 1990, p. 4; véase además Reginald E. Witt, Albinus and the History of Middle Platonism, Cambridge, 1937 (Reprint: Amsterdam 1971), 46 s.; Anonymus Prolegomena to Platonic Philosophy, Introduction, Text, Translation and Indices by Leendert G. Westerink, Amsterdam 1962, xxviii s.; Alcinous. The Handbook of Platonism, translated with an Introduction and Commentary of John Dillon, Oxford 1993, $59 \mathrm{~s}$.

19 Diogenis Laertii vitae philosophorum, hrsg. von Herbert S. Long, Oxford, 1964, V, 28.

20 Timaeus... a Calcidio translatus, ed. Jan H. Waszink, vol. IV, London, 1962, 270.

21 Ammonius In Porphyrii Isagogen sive quinque voces, ed. Adolf Busse (Commentaria in Aristotelem Graeca, 4,3), Berlin, 1891, 15.

22 Eliae In Porphyrii Isagogen et Aristotelis Categorias commentaria, ed. Adolf Busse (Commentaria in Aristotelem Graeca 18,1), Berlin, 1900, p. 31. 
virtud; la segunda se ocupa de la cura rei publicae y la tercera de la dispositio rei familiaris. ${ }^{23}$

La segunda línea comienza poco después de Boecio, con Casiodoro. ${ }^{24}$ Esta línea se prolonga en Isidoro de Sevilla ${ }^{25}$ y culmina en Hugo de San Victor. ${ }^{26}$ Según Pierre Courcelle ${ }^{27}$ existe un vinculo directo entre el comentador neoplatónico de Aristóteles Ammonio (S.V) y Casiodoro. Ello hace presumir que si bien el ingreso de la tripartición en el mundo latino se produjo casi simultáneamente a través de Boecio y de Casiodoro, sin embargo, la tradición más importante de las dos, que es la que se prolonga en forma literal desde Casiodoro hasta Hugo de San Victor, depende de la tripartición que Casiodoro recibe de Ammonio.

Esta segunda línea inaugurada por Casiodoro presenta otra clasificación de la philosophia practica, distinta de la de Boecio. Ella se reitera en forma literal hasta Hugo de San Victor. Aunque esta nueva clasificación no coincide con la de Boecio en cuanto a su formulación literaria ${ }^{28}$ sin embargo ella no introduce ningún cambio cualitativo respecto del contenido de la clasificación boeciana. Como para Boecio, tambien para Casiodoro y sus sucesores las partes de la philosophia practica siguen siendo la moralis, que trata del mos vivendi honestus y de la adquisi-

23 "Practicae vero philosophiae...triplex est divisio; est enim prima quae sui curam gerens cunctis sese erigit, exornat augetque virtutibus, nihil in vita admittens quo non gaudeat, nihil faciens paenitendum; secunda vero est quae rei publicae curam suspiciens cunctorum saluti suae providentiae sollertia et iustitiae libra et fortitudinis stabilitate et temperantiae patientia medetur; tertia vero, quae familiaris rei officium mediocri componens dispositione distribuit" (In Isagogen Porphyri commenta, ed. G. Schepss/S. Brand, CSEL 48, Vindobonae-Lipsiae, 1906, p. 9).

24 "Moralis dicitur, per quam mos vivendi honestus appetitur, et instituta ad virtutem tendentia praeparantur. Dispensativa dicitur, domesticarum rerum sapienter ordo dispositus.Civilis dicitur,per quam totius civitatis administratur utilitas" (De artibus et disciplinis liberalium litteratum, III, en Migne, PL, 70, col. 1169).

25 "Moralis dicitur, per quam vivendi mos honestus appetitur et instituta ad virtutem tendentia praeparantur. Dispensativa dicitur, cum domesticarum rerum sapienter ordo disponitur. Civilis dicitur per quam totius civitatis utilitas administratur" (Etymologiarum... Libri XX, II, 24. 16, en: Migne, $P L, 82$, col. 142).

26 "Moralis dicitur, per quam mos vivendi honestus expetitur vel acquiritur, et instituta ad virtutem tendentia praeparantur. Dispensativa dicitur, cum domesticarum rerum sapienter ordo disponitur. Civilis dicitur, per quam totius civitatis utilitas administratur" (Didascalion, II, xix, en: Migne, PL, 176, col. $760-1$ ).

27 "La dépendence directe de Cassiodore à l'égard d' Ammonius est patente, car Boèce qui [...] est aussi tributaire d'Ammonius, ne peut être la source de Cassiodore sur tous ces points" (Cfr. Pierre Courcelle, Les lettres grecques en Occident. De Macrobe a Cassiodore, Paris, 1943, p. 325). Véase tambien p. 323.

\begin{tabular}{|l|l|}
\hline \multicolumn{1}{|c|}{ Boethius } & \multicolumn{1}{|c|}{ Cassiodorus } \\
\hline $\begin{array}{l}\text { quae sui curam gerens cunctis sese erigit, } \\
\text { exornat augetque virtutibus, nihil in vita ad- } \\
\text { mittens quo non gaudeat, nihil faciens pae- } \\
\text { nitendum }\end{array}$ & $\begin{array}{l}\text { Moralis dicitur, per quam mos vivendi honestus } \\
\text { appetitur, et instituta ad virtutem tendentia } \\
\text { praeparantur }\end{array}$ \\
\hline $\begin{array}{l}\text { quae familiaris rei officium mediocri compo- } \\
\text { nens dispositione distribuit }\end{array}$ & $\begin{array}{l}\text { Dispensativa dicitur, domesticarum rerum sapi- } \\
\text { enter ordo dispositus }\end{array}$ \\
\hline $\begin{array}{l}\text { quae rei publicae curam suspiciens cuncto- } \\
\text { rum saluti suae providentiae sollertia et ius- } \\
\text { titiae libra et fortitudinis stabilitate et tem- } \\
\text { perantiae patientia medetur }\end{array}$ & $\begin{array}{l}\text { Civilis dicitur, per quam totius civitatis adminis- } \\
\text { tratur utilitas }\end{array}$ \\
\hline
\end{tabular}


ción de la virtus, la dispensativa, ocupada de la dispositio del ordo domesticarum rerum y la civilis identificada con la administratio de la utilitas civitatis.

Tanto en la línea boeciana como en la inaugurada por Casiodoro la descripción de cada una de las partes de la philosophia practica utiliza un lenguaje carente de significado político en sentido aristotélico. Ello permite establecer la primer diferencia entre la clasificación de la philosophia practica transmitida por estas dos tradiciones latinas y la clasificación aristotélica que conocerá Occidente a partir de la recepción de todos los libri morales de Aristóteles: hasta el sigio XIII Occidente conoció una philosophia practica carente de politicidad en el sentido señalado por Aristóteles. Esa pérdida de politicidad no solo es perceptible en las dos primeras partes de la philosophia practica, sino inclusive - y esto es quizá lo que sorprende - en la misma politica. En efecto, en la ethica individual o moralis el lenguaje es siempre tendencial y en él predomina el uso de expresiones desiderativas o exhortativas, pero nunca referidas a un vínculo de subordinación entre el sujeto y el objeto del acto moral (cura sui, nihil faciens paenitendum, mos honestus vivendi appetitur, moralis...instituta ad virtutem tendentia praeparantur). Lo mismo sucede en la dispensativa,definida en términos de cuidado,de preocupación o de organización de un cierto orden doméstico (dispositio, distributio). Y donde la ausencia del vínculo de subordinación entre un sujeto que manda y otro que obedece resulta aún más impactante es en la politica, caracterizada como cura rei publicae y como administratio utilitas civitatis.

La causa de esa falta de politicidad de la philosophia practica no es solo la ausencia de vínculos de subordinación. Además quedaban fuera de ella las otras notas que definen la politicidad en la tripartición aristotélica, es decir: la diferencia cualitativa entre los vínculos que definen la situación del individuo en cada una de las tres partes de la philosophia practica, la primacía lógico - ontológica de la politica sobre la ethica y sobre la oeconomica y el ordenamiento de los fines de estas dos últimas hacia la politica.

Ello permite concluir que para esta tradición: (1) las diferencias entre las tres partes de la philosophia practica son puramente cuantitativas; (2) que el tránsito desde cada una de ellas a las otras no es un pasaje problemático que requiera una caracterización teórica de la especificidad propia del vínculo de cada una de ellas; (3) ninguna de las partes de la philosophia practica tenía primacía sobre las otras. En sintesis, la situación apolítica de la philosophia practica en estas dos tradiciones latinas permite calificarlas como tradiciones en las que cada una de las partes de la philosophia practica, incluída la misma politica, aparecen totalmente despolitizadas. ${ }^{29}$

29 Dentro del ámbito latino solo Agustín presenta los vínculos domésticos y políticos en términos de subordinación: "Imperant enim, qui consulunt; sicut vir uxori, parentes filiis, domini servis. Oboediunt autem quibus consulitur; sicut mulieres maritis, filii parentibus, servi dominis" (De Civ. Dei, XIX, xiv). Sobre las relaciones entre domus y civitas: "Quia igitur hominis domus initium sive particula debet esse civitatis, omne autem initium ad aliquem sui generis finem et omnis pars ad universi, cuius pars est, integritatem refertur, satis apparet esse consequens, ut ad pacem civicam pax domestica referatur, id est, ut ordinata imperandi oboediendique concordia cohabitantium referatur ad aordinatam imperandi oboediendique concordiam civium. Ita fit, ut ex lege civitatis praecepta sumere patrem familias oporteat, quibus domum suam sic regat, ut sit paci adcommoda civitatis" (ibid., xvi). La influencia de estos textos en la baja Edad Media parece haber sido escasa; 
Esta situación no cambia en los tres autores del siglo XII que retoman la tripartición: Hugo de San Victor, Domingo Gundissalino y Guillermo de Conches.

En el tratado De divisione philosophie Gundisalino presenta una tripartición de la philosophia practica tributaria de fuentes árabes, ${ }^{30}$ pero que reitera las características de la tradición que hemos reconstruído hasta aqui. ${ }^{31}$ Es verdad que Gundisalino introduce como novedad un lenguaje más específicamente politico que caracteriza cada parte de la philosophia practica en función de un vínculo de subordinación: la politica es scientia gubernandi civitatem, ${ }^{32}$ la segunda parte de la philosophia practica es scientia regendi familiam propriam ${ }^{33}$ y la ethica individual es gubernacio sui ipsius. ${ }^{34}$ Pero Gundisalino sigue considerando cada una de esas partes en forma aislada sin establecer entre ellas vínculos de carácter sistemático y en lugar de hacer de la politica una teoría, la resuelve en una suerte de listado de virtudes que debe tener el rey. ${ }^{35}$

Y Guillermo de Conches redacta una tripartición que vuelve sobre las caracteristicas de "apoliticidad" de la tripartición de la tradición latina. Con todo, el caso de Guillermo es particularmente interesante a causa del lenguaje que utiliza para formularla. En efecto, Guillermo muestra su dependencia de la tradición iniciada por Casiodoro porque incorpora parte de la terminología de esa tradición: "dispensativa", "civilis". Pero al mismo tiempo utiliza ese vocabulario como equivalente de una nueva terminología de la philosophia practica que será la que utilizarán poco después las divisiones philosophiae de la primera mitad del siglo XIII. ${ }^{36}$ De ese modo Guillermo se presenta como un momento de tránsito entre la tradición iniciada por Casiodoro y el momento preparatorio de recepción de la Politica representado por las divisiones philosophiae.

En la última etapa de nuestra reconstrucción se encuentran las divisiones philosophiae originadas en el ambiente de los maestros de artes parisinos de la primera mitad del siglo XIII. No reconstruiré aquí exhaustivamente la concepción

véase sobre el tema S. Krüger, "Zum Verständnis der Oeconomica Konrads von Megenberg", Deutsches Archiv, 20 (1964), p. 541 s.

30 Ludwig Baur sostiene que la tripartición de Ethica Nicomachea, 1142a 9 y de Ethica Eudemia, 1281b 13 fue recibida por la escuela de comentadores de Ammonio y que de allí habria sido transmitida a los árabes. Gundisalino, que la habria recibido del mundo árabe, careceria, en consecuencia, de una dependencia respecto de la tradición latina (ćr. Dominicus Gundissalinus, De divisione philosophiae, Münster, 1903 [Beiträge zur Geschichte der Philosophie des Mittelalters, IV, 2-3] p. 310 s. y 312.).

31 “...quarum una est sciencia disponendi conversationem suam cum omnibus hominibus... secunda est sciencia disponendi domum et familiam propriam...tercia est sciencia, qua cognoscit homo ordinare modum proprium sui ipsius secundum honestatem anime sue" (cfr. ibid., p. 16).

ibid., p. 134,1.

33 ibid., p. 139,4 .

34 ibid., p. 140,4.

35 “...politica [...] est pars ethice, in quo etiam docet, quas condiciones et dispositiones naturales oportet observare in filiis regum et in aliis ad hoc, ut ille in quo inuente fuerint eligatur ad regnum, deinde illum in quo fuerint, qualiter oportet morigerari, quousque perficiatur in eo virtus regia et fiat rex perfectus" (ibid., 136).

36 "[Philosophiae] due sunt species: PRACTICA ET THEORICA. PRACTICE vero sunt tres species: ethica de instructione morum [...], echonomica id est dispensativa [...], politica id est civilis [...]" (cfr. Guillaume de Conches, Glosae super Platonem, ed. Édouard Jeauneau, Paris, 1965, p. 60). 
de la filosofia moral que presentan esas divisiones. ${ }^{37}$ Solo me referiré, en primer lugar, a las características de las divisiones que muestran su dependencia de la tradición iniciada en Casiodoro, y en segundo lugar a las características novedosas que las divisiones agregan a esa tradición.

En cuanto a las primeras, se ha señalado ${ }^{38}$ que las divisiones presentan la philosophia practica articulada en una triplex divisio sin relaciones sistemáticas entre sus partes. ${ }^{39} \mathrm{La}$ consecuencia de esa asistematicidad es el aislamiento de las partes entre sí. Esa ausencia de sistematicidad se acentúa a causa de la tendencia de las divisiones a considerar las diferencias entre sus partes solo cuantitativamente, con la consecuente pérdida de la especificidad de cada una de ellas.

Sin embargo, la característica más notable es la que las divisiones presentan como novedad respecto de la tradición: se trata de la identificación de una de las partes de la philosophia practica, la politica, con un contenido textual muy concreto: las leges del derecho romano y los decreta del derecho canónico. ${ }^{40}$ Con esa identificación las divisiones resuelven la politica en la lex y estipulan para la politica un contenido definidamente juridico, distante del carácter teórico-filosófico propio de la politica en el sistema aristotélico.

Todas estas características muestran la imposibilidad de vincular esta tradición - que comienza con Casiodoro y culmina en la primera mitad del siglo XIII con la tripartición aristotélica de la philosophia practica. ${ }^{41}$ Ello permite concluir

37 Me permito referir a dos trabajos en los que he analizado en detalle el problema: "Die Rolle der Natur in den 'Commentarii in Libros Politicorum Aristotelis' des Albertus Magnus", en: Miscellanea Mediaevalia, Bd. 21/2 (Mensch und Natur im Mittelalter), ed. Albert Zimmermann, Berlin N.York, 1992, p. 685 seg. (versión castellana: “De la politica como 'scientia legislativa' a lo politico 'secundum naturam"', en: Patristica et Mediaevalia XII [1991], p. 3/32) y "Politologische Ansichten bei den Artisten um 1230/1240. Zur Deutung des anonymen Studienplans Hs Ripoll 109", en: Theologie und Philosophie 69 (1994), p. 34 seg.

38 Georg Wieland, Ethica - Scientia practica. Die Anfänge der philosophischen Ethik im 13. Jahrhundert, Münster, 1981 (=Beiträge zur Geschichte der Philosophie des Mittelalters, NF. XXI), p. 95 seg.

39 Aunque es verdad que las divisiones philosophiae se caracterizaron en general por su asistematicidad, he procurado mostrar que en algunas de ellas existe un primer intento por superar tanto esa falencia como el aislamiento entre sus partes; véanse, por ejemplo, mis trabajos "Regimen ipsiusregimen alterius. Individuum und Gesellschaft in den Quellen des Prologus zu 'Super Ethica' des Albertus Magnus", en Miscellanea Mediaevalia, Bd. 24 (Individuum und Individualität im Mittelalter), ed. Jan A. Aertsen y Andreas Speer, 1996, p. 479 seg. (en versión castellana: "Individuo y Sociedad en el 'Prologus' del Super Ethica de Alberto Magno", en: Idade Média: Ética e Politica, organizador Luis A. De Boni, EDIPUCRS, Porto Alegre, 1966, p. 169 seg.) y "Presupuestos de la recepción de la Politica de Aristóteles", en Aristotelica et Lulliana magistro doctissimo Charles $H$. Lohr septuagesimum annum feliciter agenti dedicata (ediderunt F. Dominguez, R. Imbach, Th. Pindl et P. Walter), Martinus Nijhoff, The Hague, 1995, especialmente p. 48/51.

40 Me he ocupado del problema en "Giuridicità della 'scientia politica' nella riflessione politica degli artisti nella prima metà del secolo XII", en: LES PHILOSOPHIES MORALES ET POLIIOUES AU MOYEN AGE. MORAL AND POLITICAL PHILOSOPHIES IN THE MIDDLE AGES (Actes du IXe. Congrès international de Philosophie Médiévale), N. York-Ottawa-Toronto, 1995, Vol. I, p. 332 seg.

41 El prototipo de la philosophia practica proveniente de ese medio intelectual lo constituyeron una numerosa serie de formulaciones cuyo estereotipo aparece en un texto de Oliverus Brito: "Practica [philosophia] dividitur in monosticam, yconomicam et politicam. Monostica est quae ordinat hominem ad regimen sui, et dicitur a monos, quod est unus, et ycos, quod est scientia, quasi scientia unius ... Et hec datur ab Aristotile in Ethicis. Yconomica est que docet propriam familiam ordinare, unde yconomos grece, dispensator latine; vel dicitur ab Yconomo, huius scientie primitur inventore. Et hec a Tullio libro suo De Officiis enodatur. Politica nominatur que docet regere populos et 
que, hasta mediados del siglo XIII, en Occidente se registra una suerte de "vacío aristotélico" tanto en lo que concierne a laś relaciones entre las partes de la philosophia practica como en cuanto a la tipificación del contenido de cada una de esas partes, en especial de la politica.

Son dos los motivos que hacen de las divisiones el momento de mayor interés en el desarrollo histórico de la tripartición de la philosophia practica: en primer lugar, porque ellas constituyen el estadio inmediatamente anterior a la recepción completa de los libri morales aristotélicos; en segundo lugar porque esos libri morales actuaron directamente sobre el esquema y sobre el contenido de la philosophia practica transmitido por esas divisiones. Por ello puede afirmarse que el encuentro hacia 1265 de dos tradiciones, por una parte la tradición de la philosophia practica que comienza con Casiodoro y que presenta una suerte de esquema formal vacío, y por la otra los recientemente conocidos libri morales, constituyó un punto de inflexión que modificó radicalmente la concepción acerca de la estructura interna de la philosophia practica y la impregnó de características aristotélicas.

A todos estos datos se agrega aún otro que contribuye a agudizar el interés de esta reconstrucción. Hasta inmediatamente antes de producirse ese punto de inflexión Occidente ignoraba el contenido de la disciplina politica, pero sin embargo sabía de la existencia del texto aristotélico dedicado a ella. En efecto, conocemos tres textos que testimonian fehacientemente que en el ámbito de los maestros de artes parisinos se sabía que Aristóteles había escrito un libro acerca de la politica.

El primero es el ya mencionado tratado De divisione philosophie de Gundisalino, de mediados del siglo XII, de conocida influencia sobre los artistas. Gundisalino identifica una parte de la philosophia practica, la scientia gubernandi civitatem ${ }^{42}$ - o civilis ratio - con la politica, que habría sido tratada in libro Aristotelis. ${ }^{43}$ Más tarde, hacia mediados del siglo XIII, el texto conocido como Sicut dicit Ysaac (Ms München Clm. 14460, fol. 168 ra), primero transcripto y atribuído por M. Grabmann a Nicolás de París ${ }^{44}$ y después considerado como anónimo por C. Lafleur, ${ }^{45}$ tambien supone la existencia de la Politica, a la que se refiere como parte

civitates, unde dicitur a polis, quod est civitas vel pluralitas. Et hec traditur in legibus et decretis" (MS Oxford, Bodleian Library, Corpus Christi College, 283 fol. 151 ra-b; cfr. R. A. Gauthier, Arnoul de Provence et la doctrine de la 'Fronesis', vertu mystique suprême, en: Revue du moyen âge latin, 19 [1963], 143). Otros textos del mismo género pueden encontrarse en la nota 22 de mi trabajo “Individuo y Sociedad en el 'Prologus' del Super Ethica de Alberto Magno”, cit. supra, en nota 40. L. Baur, Dominicus Gundissalinus... (ut supra, nota 31), p. 134.

43 "Et hec quidem scientia, continetur in libro Aristotelis, qui politica dicitur, et est pars ethice, in quo etiam docet, quas condiciones et dispositiones naturales oportet observare in filis regum et in aliis ad hoc, ut ille in quo inuente fuerint eligatur ad regnum, deinde illum in quo fuerint, qualiter oportet morigerari, quousque perficiatur in eo virtus regia et fiat rex perfectus" (ibid., p. 136).

44 M. Grabmann, "Die logischen Schriften des Nikolaus von Paris und ihre Stellung in der aristotelischen Bewegung des XIII. Jahrhunderts", en: id., Mittelalterliches Geistesleben, T. I, München, 1926, p. 244.

45 C. Lafleur, Quatre introductions à la Philosophie au XIIle. siècle, Montrèal - Paris: 1988, p. 391, nota 12 . 
de la tripartición de la philosophia moralis ${ }^{46} \mathrm{Y}$ lo mismo sucede con el Ms anónimo Bruges, Biblioteque de la Ville 496, fol. 80 ra, proveniente igualmente del círculo de los artistas de alrededor de 1250; este anónimo, que recurre posiblemente a las mismas fuentes del Sicut dicit Ysaac, ${ }^{47}$ coloca a la politica como tercera parte de la philosophia moralis y se refiere a un libro en el que Aristóteles composuit scientiam de hiis. ${ }^{48}$

Un examen de estos tres textos permite sacar las siguientes conclusiones. En primer lugar, la existencia de la Politica era conocida hasta poco antes de su recepción, es decir en torno de 1250. En segundo lugar, los tres autores poseían una vaga sospecha, bastante alejada de la realidad, acerca del contenido de la Politica. En efecto, mientras Gundisalino parece asimilar la Politica al género "espejo de príncipes", los otros dos autores se muestran desorientados en relación con el contenido del libro de Aristóteles, pues no saben con certeza dónde se encuentra desarrollada la ciencia aristotélica sobre la oeconomica y la politica. Por ello oscilan entre tres posibilidades: (1) la identificación de la oeconomica y la politica con el derecho romano y canónico (leges et decreta), (2) la identificación con el De Officiis de Cicerón, y (3) la mención de un libro de Aristóteles de contenido desconocido. Y cada una de estas posibilidades es fundamentada en un tímido quidam dicunt.

Nos encontramos, pues, en una situación en la que confluyen tres datos importantes. Por un parte una tradición que comienza con Casiodoro, que llega hasta el siglo XIII y que transmite un esquema tripartito de la philosophia practica cuyo

46 "Moralis autem dividitur tripliciter quia triplex est bonum. Est enim bonum monasticum et est bonum hyconomicum et est bonum politicum. Bonum monasticum dicitur, quando aliquis debet regere seipsum tantum. Hoc modo monachus debet habere bonum monasticum, quia habet curam anime. Unde dicitur monachus a monos quod est unum et ycos quod est custos quasi custos unius sc. anime. Yconomica dicitur ab yconos quod est dispensatio quando aliquis bene scit dispensare proprie familie, propriis filiis et filiabus, famulis et famulabus. Politica dicitur a 'polis', quod est civitas, secundum quod huius prelati dicuntur regere civitates ut prepositi et alii. Due ultime partes scilicet yconomica et politica, sicut quidam dicunt, traditur in legibus et decretis, alii dicunt, quod traditur a Tullio in libro de officiis. Alii dicunt, quod Aristoteles fecit in lingua arabica quandam scientiam de hoc, que nobis adhuc non est translata" (cfr. Grabmann, ut supra, nota 45, p. 245-6).

47 La similitud entre ambos textos ha sido señalada por R. A. Gauthier en "Amoul de Provence et la doctrine de la fronesis, vertu mystique suprême", en Revue du moyen âge latin, 19 (1963) p. 141. Lafleur (ut supra, nota 46, p. 391) retoma esa hipótesis.

48 "Tunc accedendum est ad divisionem moralis philosophie vel scientie. Que, cum sit de bono ut dictum fuit, triplex autem est bonum, scilicet monosticum, yconomicum, politicum. Monosticum dicitur illud bonum per quod aliquis regit et ordinat se ipsum tantum, ut sit in monachis et religiosis viris qui non habent aliquam curam nisi sui ipsius tantum, et in pluribus laycis; unde dicitur monosticum, a monos quod est unum, et custos, quasi scientia de custodia unius. Aliud autem est bonum quo aliquis se et sibi coniunctas personas regit ut pater familias se et suam familiam,et hoc est bonum yconomicum; ycos idem est factor vel dispensator, talis dispensat familie sue. Tertium est bonum politicum, a polis quod est civitas, quo aliquis regit se et sibi coniunctos et totam civitatem sive plures, scilicet civitates. Secundum bonum est in hominibus maritatis, tertium est in principibus terre et prelatis. De bono yconomico et politico non habemus aliquam scientiam, sed ut dicunt aliqui, de istis determinatur in legibus et decretis, vel, secundum alios, in quodam libro a Tullio composito, aut etiam, secundum alios, Aristoteles composuit scientiam de hiis, sed nondum est adhuc translata nobis in latinum" (crr. Gauthier, ut supra, nota 48, p. 142). 
contenido muestra que su origen no es aristotélico. Por otra parte la tradición de las divisiones philosophiae de la primera mitad del siglo XIII que retoma ese esquema con todas sus caraacterísticas y que identifica la disciplina politica con un contenido jurídico: leges et decreta. Y por fin tenemos textos de autores que testimonian que inmediatamente antes de conocerse el texto de la Politica se sabía que ella existía, aunque no se sabía su contenido. Paradigma de esta tercera situación es el siguiente texto:

\footnotetext{
"De bono yconomico et politico non habemus aliquam scientiam, sed ut dicunt aliqui, de istis determinatur in legibus et decretis, vel, secundum alios, in quodam libro a Tullio composito, aut etiam, secundum alios, Aristoteles composuit scientiam de hiis, sed nondum est adhuc translata nobis in latinum." 49
}

La expresión "de bono yconomico et politico non habemus aliquam scientiam" es una clara expresión de la situación que he presentado al comenzo de esta ponencia, i.e.: Occidente poseía el esquema de la philosophia practica y conocía el nombre de las disciplinas que la integran, pero carecía de su contenido científico. Fácil es percibir qué fue lo que sucedió tan pronto como se conoció en Occidente el texto de la Politica. Tanto los comentarios a la Politica como los tratados políticos de inspiración aristotélica sustituyeron, en el esquema transmitido a partir de Casiodoro, el contenido jurídico de la disciplina politica por el contenido que Aristóteles le atribuye en la Politica.

Un ejemplo de la rápida inclusión de los contenidos aristotélicos en el esquema de la philosophia practica puede percibirse en el tratamiento que hace Alberto Magno del problema de la sistematicidad y de la unidad que reune a las tres disciplinas que integran la philosophia practica..$^{50}$ Alberto se ocupa del mismo problema, primero en su comentario a la Ethica Nicomachea (=Super Ethica) y después en su comentario a la Politica.

En el Super Ethica, escrito entre 1248 y 1252, i.e. bastante antes de conocer la Politica, Alberto procura responder la pregunta utrum disciplina moralis sit una ${ }^{51}$ utilizando recursos propios, i.e. recursos no específicamente aristotélicos, que no están presentes en el texto de la Ethica. Allí construye una argumentación que unifica a las tres ciencias, primero de arriba hacia abajo, porque la ethica individualis ofrece principios a la oeconomica y a la politica, y luego de abajo hacia arriba, porque la politica se ocupa de hacer efectiva la aplicación de esos principios de la ethica individualis. Bien diferente es la respuesta que ofrece Alberto al mismo problema en su comentario a la Politica, donde asume la posición del mismo Aristóteles y apela al argumento aristotélico que atribuye a la politica una función conglutinante de los fines de las restantes ciencias prácticas. ${ }^{52}$

49 Como nota 49.

50 Me he ocupado extensamente del problema en: "Die Rolle der Natur in den 'Commentarii in Libros Politicorum Aristotelis' des Albertus Magnus", (versión castellana: "De la politica como 'scientia legislativa' a lo politico 'secundum naturam'"), ambos citados supra, nota 38.

51 Cfr. Alberti Magni Super Ethica. Commentum et Quaestiones, en: Alberti Magni Opera Omnia, T. XIV, Pars I-II, ed. W. Kübel, Münster, 1968 ss., p. 2 a.

52 "Principalissima autem omnium, est quae omnes alias complectens communicationes"; "Omres autem complectens, civitas est communicatio politica" (cfr. D. Alberti Magni... Commentarii in octo libros Politicorum Aristotelis [ed. A. Borgnet], Paris, 1891, p. 8 a). 
Se trata, pues, de un caso evidente del ingreso en el esquema de la philosophia practica del contenido teórico de una ciencia que Alberto conocía de nombre, pero cuyo contenido todavía desconocía cuando escribía el Super Ethica. Tan pronto como conoce el texto de la Politica Alberto lo incorpora al esquema de la philosophia practica y lo utiliza para resolver los mismos problemas que antes había tratado si ella.

El esquema de la philosophia practica es solo un ejemplo de la función ejercida por los esquemas del saber como organizadores de ramas del conocimiento científico y como integradores de contenidos científicos aún no incorporados al esquema del saber. Cary Nedermann ha hecho referencia a la función de esos esquemas en los siguientes términos: "The triumph of Aristotelianism in moral and political study after the mid-thirteenth century may perhaps be measured not only in the broad acceptance of various doctrines proposed by Aristotle but especially in the pervasiveness of the Aristotelian scheme of practical knowledge and its implications." 53

53 Cfr. Cary J. Nederman, 'The Meaning of 'Aristotelianism' in Medieval Moral and Poliical Thought”, en Joumal of the History of Ideas 57 (1996), p. 580. 